\title{
Article
}

\section{Optimizing Age-Structured and Risk-Based Mass Drug Administration Against Soil-Transmitted Helminthiasis in Ghana Using Cost-Effectiveness Analysis}

\author{
Mugdha Thakur ${ }^{1}$, Rasheeda Mohammed ${ }^{2, a}$, Anuj Mubayi ${ }^{3, *}$ \\ 1 mthakur4@asu.edu \\ 2 ramniham92@gmail.com \\ 3 amubayi@asu.edu \\ 1, 2,3 School of Human Evolution and Social Change, Simon A. Levin- Mathematical, Computational and \\ Modelling Sciences Center, Arizona State University, AZ 85287 \\ a Harvard/MGH Center on Genomics, Vulnerable Populations, and Health Disparities, Massachusetts \\ General Hospital, Boston, MA 02114 \\ * Corresponding author: amubayi@asu.edu; Tel.: +1-480-965-2115
}

\begin{abstract}
Soil-transmitted helminthiasis (STH), a neglected tropical disease (NTD) remains a major health problem all over the world including Ghana, which has STH prevalence of $25.4 \%$. To control the disease, the government of Ghana currently concentrates on implementing mass drug administration (MDA) efforts focusing only among school-aged children. However, various studies have suggested that focusing on only a specific group for MDA may not be cost-effective. Moreover, some adults such as teachers and school-workers spend large fraction of their time with children, who shed more parasite in environment due to unhygienic behavior, and thus have a higher risk of getting infected as compared to other adults. In this study we use a mathematical model to evaluate age-structured and risk-based policies for implementing MDA while capturing transmission dynamics of STH in Ghana. A cost model was developed that included various costs related to MDA to study cost-effectiveness of current policies of MDA in Ghana against novel policies to control STH in Ghana. We carry out analysis for five different scenarios - I: no MDA (baseline), II: current MDA policy (focusing children) in Ghana, III: MDA for different age groups (adults and children groups) for unlimited budget, IV: MDA for different age groups with limitations of number of individuals treated, and, V: MDA for different groups based on their risk of getting infected (adults school workers (high-risk group), adults non-school workers and children groups). Our results suggest that it might be more cost-effective to allocate treatment through MDA to at least some proportion of adults along with children. In case of unlimited budget, the best strategy in Scenario IV would be to treat approximately $22 \%$ of adults and approximately $45 \%$ of children. The most cost-effective among the 5 scenarios is suggested through scenario V, where high-risk adults group and children are provided MDA at higher level than low-risk adults. In conclusion, age-structured and risk-based allocation of treatment and resources is crucial to reducing STH load in developing countries.
\end{abstract}

Keywords: helminthiasis dynamics in Ghana, preventive chemotherapy, school-aged children, high-risk adults, neglected tropical diseases, age-structure, infection risk, cost analysis

\section{Introduction}

Epidemiology of soil-transmitted helminthiasis: Globally, Soil-transmitted helminthiasis (STH) is considered one of the major public health problems. As many as 300 million people suffer from severe morbidity due to helminths infection annually resulting in about 150,000 deaths annually [1]. In Ghana the prevalence of STH is $25.4 \%$. Soil-transmitted helminths are of three 
species: roundworm (Ascaris lumbricoides), whipworm (Trichuris trichiura) and the hookworms (Necator americanus and Ancylostoma duodenale). Roundworm affects about 1.1 million people worldwide; hookworm and whipworm affect between 740,000 to 800,000 worldwide (Centers for Disease Control and Prevention, 2013) with the highest prevalence in sub-Saharan Africa [2]. The prevalence of hookworm in the middle belt of Ghana is 45\% [3]. People infected with soil-transmitted helminthiasis or intestinal worms show symptoms such as diarrhea, general abdominal pains, weakness and in the case of hookworm, blood loss which can result in severe anemia. All three species of helminths thrive in warm and temperate regions and particularly in places where sanitation and hygiene are poor. Environmental factors such as inaccessibility to safe drinking water, improperly cooked foods, limited or lack of toilets are examples of difficulties faced by millions of people in Ghana and these environmental factors have immensely contributed to the prevalence of soil-transmitted helminths in Ghana and around the world. Even though it is curable and eliminable, it remains a constant health problem in the lives of many young people in Ghana both old and young. Around the world, about a quarter of the world's population is affected by STH thereby causing significant disability to these affected people.

Life cycle of helminths worms: Ingestion of helminths happens upon consumption of food that is contaminated by helminth worms or when hands or food with contaminated dirt are put in mouth without washing [4]. Roundworm and whipworm are transmitted by ingestion of worm eggs which then settle and grow in the intestines of humans. Hookworm (for example Anclostoma duodenale) are transmitted by the penetration of filariform (a larvae form) through the skin and then enters the blood stream to travel and mature in the small intestine. The adult worms then release eggs into the soil (or environment) through improperly disposed human feces [5]. As shown in Figure 1. .Life cycle and mode of transmission of helminths (hookworm) [4,6].Figure 1, the worms are infective only when they enter the human body (either by ingestion or by penetration through skin) in the filariform larva stage.

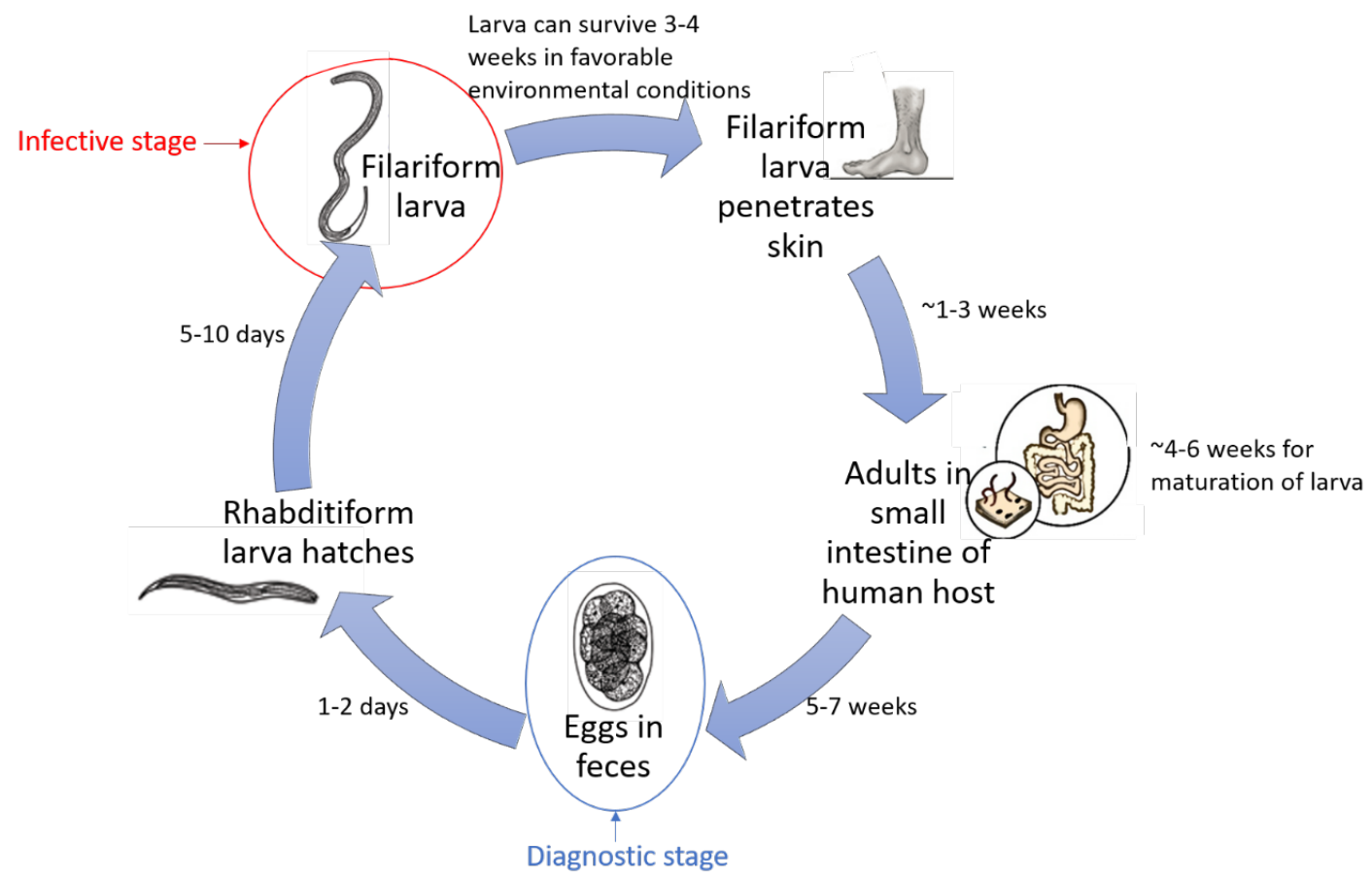

Figure 1. .Life cycle and mode of transmission of helminths (hookworm) [4, 6].

Soil-Transmitted Helminthiasis in Ghana: The prevalence of soil-transmitted helminthiasis is widespread across all 10 regions of Ghana however, some forms of it are less prevalent in some regions than in others. Mass drug administration (MDA), the delivery of essential medicines as a preventive measure where populations or sub-populations are offered medicine irrespective of their 
health condition or individual diagnosis [7], is essential for decreasing pathogen levels in the population, reducing and interrupting the transmission of the disease, thereby controlling and eliminating NTDs like Lymphatic Filariasis, Trachoma, Onchocerciasis, Schistosomiasis and STH [8], [9]. MDA is implemented in Ghana since 2000. However, this disease is still recurring among vulnerable populations especially people in rural areas. People living in urban areas in Ghana are not spared of the dangerous effects of this disease as they too are affected because of infrastructural problems such as poor city planning which reduces their accessibility to clean water and toilets.

In line with following WHO's guidelines on mass drug administration, 7 million people in Ghana have been targeted for MDA (also called as preventive chemotherapy) through the use of albendazole or mebendazole - two drugs used against STH. MDA is a key strategy currently being used to control the spread of STH by Ghana's Ministry of Health. The goals of the ministry are "to treat all school-aged children in endemic areas and to reduce STH related morbidity to levels of no public health significance" [10]. School-based (environments where STH is most likely to be transmitted) mass drug administration, health education and awareness, use of clean water and the monitoring and evaluation of progress of MDA are some of the interventions used by the Ministry of Health in Ghana to curb the disease. The target group for MDA in Ghana is children, particularly school-aged children (5-14 years). Other age groups that are vulnerable to the disease are the pre-school aged children (0-4 years) and high-risk adults (>15 years) but these age groups are not targeted in current MDA efforts [10]. Although there are positive indicators leading to the gradual achievement of these goals for instance through Water, Sanitation, Hygiene (WASH) programs, (WASH and the Neglected Tropical Diseases, 2013) there is little to no information on dynamics of STH that can examine variations of coverage within the total population that could boost MDA intervention efforts against the disease with changing environmental and political conditions in Ghana. This paper therefore seeks to contribute to research on soil-transmitted helminthiasis in Ghana by using a comprehensive mathematical framework to estimate optimal coverage of MDA between the children, high-risk adult and low-risk adult populations that could eventually eliminate STH from the country. According to Smits (2009) "at current suboptimal coverage rates, programs may need prolongation for an extended period, increasing costs and undermining sustainability" [11]. This means that current MDA programs are unsustainable due to less effective coverage policies, which could result in reinfection within and between groups.

Mixing of populations: In this study, we refer to the terms homogeneous mixing and heterogeneous mixing of the populations. In the context of the scenarios, homogeneous mixing would be referred to the assumption that all adults mix or spend time equally with all the children in the population. Heterogeneous mixing of adults is referred to the assumption that some class of adults, like teachers or school-workers, mix or spend time disproportionately more with the children as compared to the rest of the adults.

Risk-based categorization: Scenario of heterogeneous mixing is discussed due to the unhygienic behavior of the children which leads to the introduction of comparatively more parasites in the environment. The teachers are considered high-risk adult population since their exposure to parasites on the surfaces like desks, chairs, doors etc. is higher due to sharing the space with children. Therefore, they are also more likely get the STH infection due to penetration or ingestion of these parasites.

Age-structured categorization: The population in consideration is divided into two groups based on age: Children ( 0 to 14 years) and Adults (15 years and above). This classification is based on the difference in hospitability of host body for growth and reproduction of parasites and in the probability of contact with contaminated environment. For example, higher likelihood of children playing bare-feet in the soil which may be contaminated and increasing susceptibility to infection due to waning immune-competence with age.

Health Metrics: Understanding the impact of health programs or the lack thereof, requires measurable outputs. Policy-makers need health metrics to evaluate the effectiveness health interventions. This informs healthcare decision-making and strengthens health systems. Health metrics are necessary in identifying the best health intervention strategies to control and eliminate 
diseases. Knowing the type of health metrics to use informs the decision structures that can lead to a healthier population.

Goal of the study: Although, hookworm infection is common in children, the prevalence and infection intensity are highest in adults [12]. Therefore, the goal of this work is to find the optimal coverages of MDA for children and adults for STH in Ghana via construction of a novel model for transmission of STH for different coverages for MDA. Using the hybrid model that involves dynamical and cost models, examine cost-effectiveness of different MDA strategies with change in prevalence to test the hypothesis that it is cost-effective to include adults along with children in MDA in Ghana. The different strategies would be testing cost-effectiveness of current policy and the proposed policy of including adults against the cost incurred against the prevalence when there was no MDA for STH in Ghana. We also observe how the cost distributions for different coverages change under different assumptions for budget allocated.

This paper is a novel work which examines community-wide mass drug administration by finding optimal coverage of MDA between adults and children in Ghana. In particular, we aim to focus on these research questions: (1) How does the dynamics of STH in Ghana change when different MDA coverages in children and adult are considered? (2) What are the optimal percentages of coverage between adults and children that could reduce prevalence of STH in Ghana and are cost-effective? (3) How do these optimal coverages change if the MDA coverage is for children and high-risk adults only?

\section{Materials and Methods}

The anti-helminthic treatment required can be quantified from the quantification of the worm load in the host [13]. Mathematical transmission model of the mean worm burden adapted from Anderson and May's work on helminths in 1991 [14] is used in this research to find optimal percentages of coverage of MDA between adults and children. As part of reducing prevalence of $\mathrm{STH}$, "the goal of the World Health Organization is to reduce the prevalence of moderate and heavy infections with soil transmitted helminths in preschool and school aged children to below $1 \%$ by 2020" [15]. We first set up the baseline case where there is no MDA and prevention are conducted solely by treating the infected people.

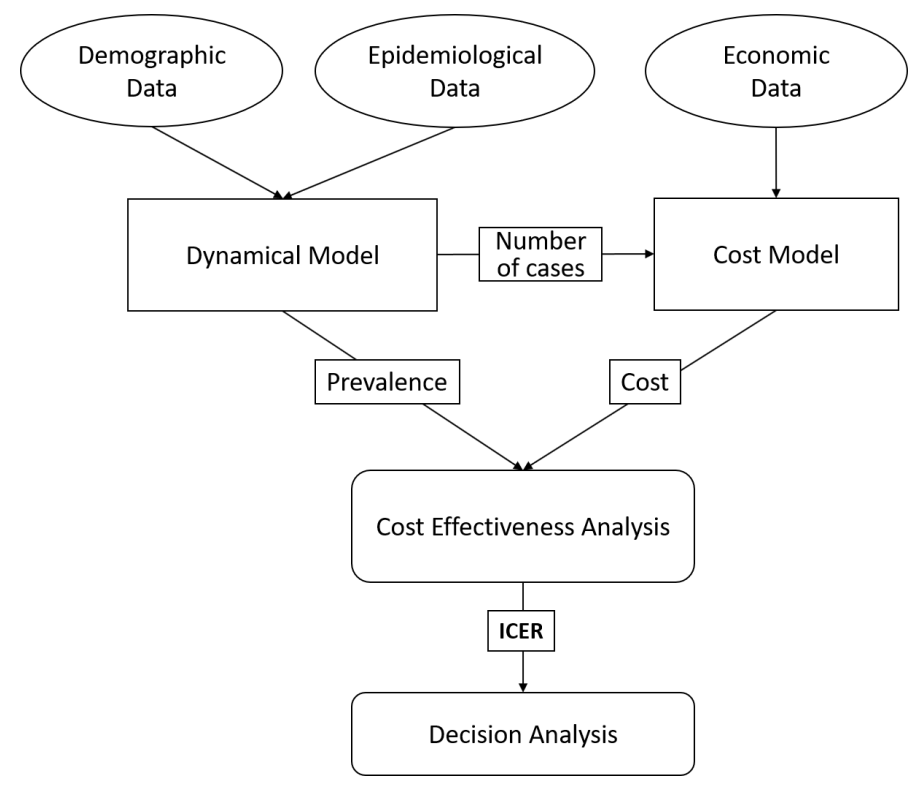

Figure 2. Flowchart of analysis.

Then we evaluate the current strategy where only $80 \%$ of school-aged children are covered. We first simulate initial prevalence and mean worm burden to calculate for change in mean worm burden and change in prevalence over time ( $t$ in years) in four case setups. To analyze for 
cost-effectiveness of various strategies, we use the prevalence and cost values from before MDA i.e. the baseline case. The structure of analysis is summarized in Figure 2.

\subsection{Homogeneous mixing of adults with children}

\subsubsection{Dynamical model}

Mean worm burden is the number of worms an individual host carries. The dynamics of worms in hosts and environment and how MDA reduces the mean worm burden in the system is shown in Figure 3. This is modeled using the following equation [16]

$$
\frac{d M}{d t}=\mu M\left(R_{0} f(M, k, z)-1\right)
$$

where $R_{0}$ is the basic reproduction number defined as the successful transfer and maturation of a worm in host upon reproduced by an adult worm in a host body, $k$ is the negative binomial aggregation parameter, $\mu$ is the per capita mortality rate of the worms in host. Fecundity, $f(M, k, z)$, is the "measure of the number of off-spring produced by one organism over time" (Biology Dictionary, 2013), is modeled as follows:

$$
\mathrm{f}\left(M(t), k_{p} \mathrm{z}\right)=\left(1+\frac{M(l)(1-z)}{k}\right)^{-(k+1)}
$$

where $z=e^{\gamma}$ represents the impact of density-dependent egg production $(\gamma)$ and is set to 0.98 in this paper [17].

To incorporate the effect of MDA in the dynamics of the worm transmission for the two age-structures (children and adults) with the assumption of homogeneous mixing, we contructed a modified model as shown in Figure 3 using following equations. The change in mean worm burden in children is given by

$$
\frac{d M_{1}}{d t}=\beta_{1} R o f\left(M_{1}, k_{1}\right) M_{1}\left(1-\frac{X_{1}}{L}\right)-\mu M_{1}
$$

and the change in mean worm burden in adults is given by

$$
\frac{d M_{2}}{d t}=\beta_{2} R o f\left(M_{2}, k_{2}\right) M_{2}\left(1-\frac{X_{2}}{L}\right)-\mu M_{2}
$$

where $X_{1}$ and $X_{2}$ are the coverages of MDA in children out of total population of children $\left(N_{1}\right)$ and in adults out of total population of adults $\left(N_{2}\right)$ respectively. $L$ is the normalizing factor given by

$$
L=\frac{\beta_{1} M_{1}+\beta_{2} M_{2}}{M_{1}+M_{2}}
$$

and $R_{0}$ is the basic reproduction number defined as the average number of parasites reproduced by a worm in an infective host and successful transmission and maturity in a susceptible host. In this system we are only considering female worm population since only females are capable of reproduction. 
Table 1. Variables used in dynamical model.

\begin{tabular}{cc}
\hline Variables & Definitions \\
\hline $\mathrm{M}_{1}$ & Mean worm burden in children \\
$\mathrm{M}_{2}$ & Mean worm burden in adults \\
$\mathrm{M}_{3}$ & Disease prevalence in children \\
$\mathrm{P}_{1}$ & Disease prevalence in adults \\
$\mathrm{P}_{2}$ & Disease prevalence in high-risk adults \\
$\mathrm{P}_{3}$ & Number of infected children \\
$\mathrm{I}_{1}$ & Number of infected adults \\
$\mathrm{I}_{2}$ & Number of infected high-risk adults \\
$\mathrm{I}_{3}$ & Fecundity of worms \\
$\mathrm{f}(\mathrm{M}, \mathrm{k})$ &
\end{tabular}

Using the mean worm burden from the dynamical system, the prevalence is calculated using [18]:

$$
P(M(t), k)=1-\left(1+\frac{M(t)}{k}\right)^{-k}
$$

As summarized along with other variables in the dynamical model in 
Table 1, $P_{1}$ is the prevalence in children and is given by

$$
P_{1}=1-\left(1+\frac{M_{1}}{k_{1}}\right)^{-k_{1}}
$$

and $P_{2}$, the prevalence in adults, is given by

$$
P_{2}=1-\left(1+\frac{M_{2}}{k_{2}}\right)^{-k_{2}} .
$$

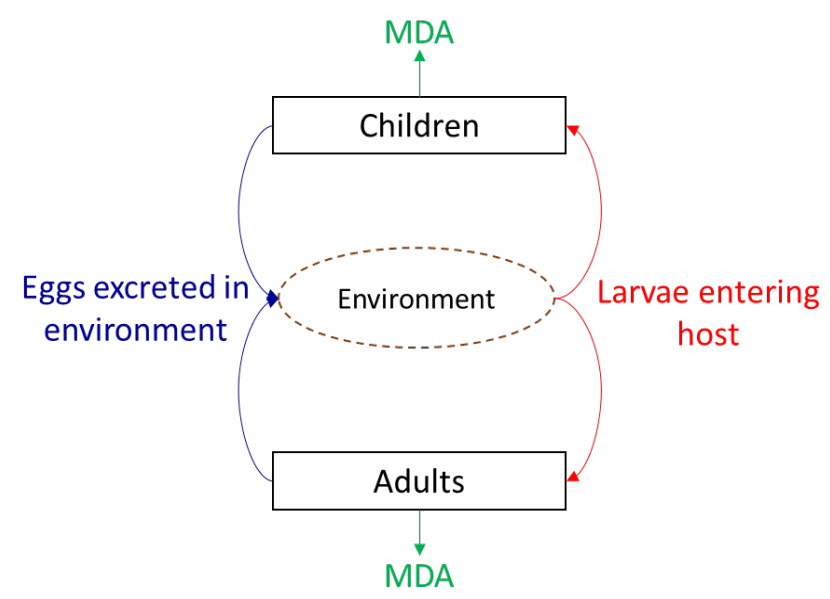

Figure 3. Flowchart of dynamical model.

\subsubsection{Cost model}

Given a coverage for MDA in the children and adult populations, the cost for delivering MDA per individual is calculated using the costs for drugs (treatment), human resources and delivery (for example transportation) as follows:

Cost of implementing MDA in children is:

$$
C_{1}=C_{H}+C_{C} / X_{1}
$$

Cost of implementing MDA in adults is:

$$
C_{2}=C_{H}+C_{A} / X_{2}
$$

Here, $C_{C}$ and $C_{A}$ are defined as the time value of staff or how much it would cost to pay an individual to administer drugs to a patient (child and adult respectively). Note that these costs $\left(C_{1}\right.$ and $C_{2}$ ) are calculated only when there is at least some MDA coverage and hence are well-defined.

We assumed as the coverage is increased, the cost of transportation per patient decreases. Because of the effect of economies of scale which means that as the number of people treated increases, the cost per treatment decreases [19]. This scale is done using the proportions $X_{1}$ and $X_{2}$ as shown above.

Therefore, the total cost of treating children and implementing MDA in children with $\mathrm{X}_{1}$ coverage is the sum of the cost of drug needed to treat infected children accessing health-care and the cost of implementing MDA at a certain percentage of coverage of the total population in children. This is shown as:

$$
T_{1}=C_{D} \cdot I_{1}(t)+\left(C_{D}+C_{1}\right) \cdot X_{1} N_{1}
$$

where number of infected children $I_{1}(t)=P_{1}(t) \cdot N_{1}$ is obtained from the dynamical model above. 
Similarly, the total cost of treating children and implementing MDA in adults with $\mathrm{X}_{2}$ coverage is the sum of the cost of drug needed to treat infected adults and the cost of implementing MDA at a certain percentage of coverage of the total population in children.

$$
T_{2}=C_{D} \cdot I_{2}(t)+\left(C_{D}+C_{2}\right) \cdot X_{2} N_{1}
$$

where the number of infected adults $I_{2}(t)=P_{2}(t) N_{2}$.

Therefore, the total cost of treating and implementing MDA strategically with $\mathrm{X}_{1}$ coverage in children and $\mathrm{X}_{2}$ coverage in adults is

$$
T=T_{1}+T_{2}
$$

\subsubsection{Incremental Cost-Effectiveness Ratio (ICER)}

The Incremental Cost-Effectiveness Ratio (ICER) in this analysis is the "difference in cost between two possible interventions, divided by the difference in their effect" [5]. This model uses the outputs of the earlier two models: Dynamic model (Section 2.1.1) and Cost model (2.1.2). ICER is calculated as the additional cost incurred against the averted prevalence:

$$
\text { ICER }=\frac{T-T_{0}}{P_{0}-P}
$$

Here, numerator is the total increment in cost due to MDA and as the baseline, we use the costs and values where $\mathrm{X} 1=\mathrm{X} 2=0$ i.e. no MDA, just treatment. Therefore,

$$
T_{0}^{*}=C_{D}^{*} I_{0}
$$

where $I_{0}=I(0)$ is the total number of people infected in the absence of MDA.

The denominator represents the total benefit i.e. total decrement in the prevalence of STH in population. The total prevalence $P$ is given by

$$
\frac{P_{1} N_{1}+P_{2} N_{2}}{N_{1} \| N_{2}}
$$

and the baseline used, P0, is the prevalence of STH before MDA.

\subsubsection{Strategies for MDA}

\begin{tabular}{|c|c|c|c|}
\hline Parameters & Definitions & Value Estimates & Source \\
\hline$\beta_{I}$ & Contact ratio of children & 1.11 & [16] \\
\hline$\beta_{2}$ & Contact ratio of adults & 0.56 & [16] \\
\hline$\mu$ & $\begin{array}{l}\text { Average mortality rate of worms } \\
\qquad\left(\text { years }^{-1}\right)\end{array}$ & 0.545 & [17] \\
\hline$R_{0}$ & Basic reproduction number & 2 & {$[20]$} \\
\hline$k_{1}$ & $\begin{array}{l}\text { Negative binomial dispersion of } \\
\text { infection in children }\end{array}$ & 0.0135 & $\begin{array}{c}\text { Calculated from } \\
\text { prevalence of STH in } \\
\text { children }\end{array}$ \\
\hline
\end{tabular}

The purpose of this work is to get an insight on the effects of different MDA strategies with age-structure so that soil-transmitted helminths can be controlled faster and more efficiently and still be cost-effective. As summarized in Table 4, the different strategies are

Table 2. Parameter definitions and estimates for the dynamical model. 


\begin{tabular}{|c|c|c|c|}
\hline$k_{2}$ & $\begin{array}{l}\text { Negative binomial dispersion of } \\
\text { infection in children }\end{array}$ & 0.089 & $\begin{array}{c}\text { Calculated from } \\
\text { prevalence of STH in } \\
\text { children }\end{array}$ \\
\hline Z & Density-dependent coefficient & 0.98 & [17] \\
\hline$X_{1}$ & $\begin{array}{c}\text { Proportion of MDA coverage in } \\
\text { children }\end{array}$ & {$[0,1]$} & Varied for optimization \\
\hline$X_{2}$ & $\begin{array}{l}\text { Proportion of MDA coverage in } \\
\text { adults }\end{array}$ & {$[0,1]$} & Varied for optimization \\
\hline$X_{3}$ & $\begin{array}{c}\text { Proportion of MDA coverage in } \\
\text { high-risk adults }\end{array}$ & {$[0,1]$} & Varied for optimization \\
\hline$N_{1}$ & Total population of children in Ghana & 10185572 & {$[21]$} \\
\hline$N_{2}$ & Total population of adults in Ghana & 16408613 & {$[21]$} \\
\hline$N_{3}$ & $\begin{array}{l}\text { Total population of high-risk adults } \\
\text { (teachers) in Ghana }\end{array}$ & $\mathrm{N}_{1} / 32.83$ & $\begin{array}{l}\text { UNESCO Institute for } \\
\text { Statistics }\end{array}$ \\
\hline
\end{tabular}

Scenario I: This is the baseline case in which there is no MDA at all. That is $X_{1}=X_{2}=0$. The cost effectiveness of all the other strategies will be tested against this case.

Scenario II: To evaluate the current MDA strategy in Ghana, we examine the dynamics of change in prevalence when only children are (80\% of coverage) targeted for mass drug administration. This means that there is no coverage of adult in mass drug administration so $X_{2}$ is kept at 0 .

Scenario III: This case includes implementation of MDA to adults along with the children. The assumption behind this strategy is that the resources are unlimited and hence it is possible to treat the whole population as one of the cases. Thus, this strategy is analyzed for all the combination of coverages for children and adults in $0 \%$ to $100 \%$ for least ICER.

Scenario IV: In this case, to make the previous strategy more realistic we put a constraint on the number of individuals receiving MDA. Therefore, in this case too, the adults are also covered in MDA, but we fixed the total number of individuals treated to be equivalent to the number of individuals in MDA in the current policy (Scenario II) due to budget constraints. Thus ensuring that the total number of individuals covered under MDA becomes $0.8 N_{1}$ individuals. The range of children covered in MDA $\left(X_{1}\right)$ is from $0 \%$ to $80 \%$, but the corresponding coverage in adults is calculated as

$$
X_{2}=\frac{0.8 N_{1}-X_{1} N_{1}}{N_{2}}
$$

Table 3. Variable definitions and estimates for the cost model.

\begin{tabular}{cccc}
\hline Variable & Quantities (per patient) & Value Estimate & Source \\
\hline $\mathrm{C}_{\mathrm{D}}$ & Cost per unit drug: Albendazole & $\$ 0.56$ & \\
& & & \\
$\mathrm{C}_{\mathrm{c}}$ & $\begin{array}{c}\text { Cost of transportation for } \\
\text { implementing MDA in children }\end{array}$ & $\$ 0.54$ &
\end{tabular}




$\begin{array}{cccc}\mathrm{C}_{\mathrm{A}} & \begin{array}{c}\text { Cost of transportation for } \\ \text { implementing MDA in adults }\end{array} & \$ 1.62 & \begin{array}{c}\text { Using the 3x multiplier effect } \\ \text { from Lo et. al 2015 }\end{array} \\ \mathrm{CH}_{\mathrm{H}} & \text { Cost of human resources } & \$ 0.05 & \text { [24] }\end{array}$

To identify the most cost-effective strategy in each scenario, ICERs are calculated for every combination of coverages as given above for the steady state prevalence and corresponding cost. The combination with least ICER is deemed to be the optimal strategy for MDA in that scenario.

Table 4. Summary of strategies for MDA in Ghana.

\begin{tabular}{|c|c|c|c|}
\hline \multirow[t]{2}{*}{ Scenario } & \multirow[t]{2}{*}{ Description } & \multicolumn{2}{|c|}{ Coverage } \\
\hline & & Children $\left(X_{1}\right)$ & Adults $\left(X_{2}\right)$ \\
\hline I: Baseline & No MDA & 0 & 0 \\
\hline II: Current policy & MDA only to children & $80 \%$ of children ${ }^{1}$ & 0 \\
\hline $\begin{array}{l}\text { III: Assuming } \\
\text { unlimited resources }\end{array}$ & $\begin{array}{l}\text { MDA to both children and } \\
\text { adults }\end{array}$ & {$[0,100] \%$ of children } & {$[0,100] \%$ of adults } \\
\hline $\begin{array}{c}\text { IV: Constraint on } \\
\text { number of } \\
\text { individuals in MDA }\end{array}$ & $\begin{array}{l}\text { MDA to both children and } \\
\text { adults but total number of } \\
\text { individuals is fixed } \\
\qquad\left(=0.8 \mathrm{~N}_{1}\right)\end{array}$ & {$[0,80] \%$ of children } & {$[0,49.67] \%$ of adults } \\
\hline
\end{tabular}

${ }^{1}$ This is referred to as the capacity of the number of individuals under MDA in Ghana. 


\subsection{Heterogeneous mixing of adults with children}

Heterogeneous mixing of adults with children may result in disproportionate risk of infection for adults. We broadly consider two types of cases, scenarios in which each adult experience same risk with children (Table 4; Section 2.1) and scenarios in which some adults (e.g., teachers and school workers; Scenario V) are at high-risk for acquiring infection as they spend more time with children. In the last case, we consider a separate class of adults, like teachers, who mix with children more than other adults as described under heading Mixing of populations in Section 1.

\subsubsection{Dynamical model}

For this case the dynamical model becomes:

$$
\begin{aligned}
& \frac{d M_{1}}{d t}=\beta_{1} R_{0} f\left(M_{1}, k_{1}\right) M_{1}\left(1-\frac{x_{1}}{L}\right)-\mu M_{1} \\
& \frac{d M_{2}}{d t}=\beta_{2} R_{0} f\left(M_{2}, k_{2}\right) M_{2}-\mu M_{2} \\
& \frac{d M_{3}}{d t}=\beta_{1} R_{0} f\left(M_{3}, k_{2}\right) M_{3}\left(1-\frac{x_{3}}{L}\right)-\mu M_{3}
\end{aligned}
$$

where the normalizing factor $L$ has changed to

$$
L=\frac{\beta_{1} M_{1}+\beta_{2} M_{2}+\beta_{1} M_{3}}{M_{1}+M_{2}+M_{3}}
$$

and the calculation of prevalence incorporates the prevalence of this new class too as follows

$$
P=\frac{P_{1} N_{I}+P_{2} N_{2}+P_{3} N_{3}}{N_{1}+N_{2}+N_{3}}
$$

In this system, the mean worm burden of the high-risk adults $\left(M_{3}\right)$ is dependent on the contact ratio $(\beta)$ similar to the children since the high mixing is assumed to be in the schools while the fecundity is similar as the adults. Alternatively, the contact ratio can also be a weighted average of children's and adults' contact ratios. Here, $X_{3}$ is the proportion of the number of high-risk adults in MDA out of the total number of high-risk adults (N3) and no low-risk adults will be covered under MDA. To calculate the number $\mathrm{N}_{3}$, we use the student-to-teacher ratio which was 32.83 in 2016 according to the United Nations Educational, Scientific, and Cultural Organization (UNESCO) Institute for Statistics ${ }^{1}$.

\subsubsection{Cost model}

Consequently, the updated structure of costs would be

$$
C_{1}=C_{3}=C_{H}+C_{C} / X
$$

where $C_{1}$ and $C_{3}$ are the costs of delivery of MDA per individual in the two groups children and high-risk adults respectively. In this scenario, the reduction in cost of delivery was scale by the total proportion of individuals under MDA given by the following relation

$$
X=\begin{gathered}
X_{1} N_{1}+X_{2} N_{3} \\
N_{1}+N_{3}
\end{gathered}
$$

Note that the cost of transport for high-risk adults is same as children since they will also be given the preventive chemotherapy at school. Therefore, the total costs per group are

${ }^{1}$ Source: https://www.indexmundi.com/facts/ghana/pupil-teacher-ratio 


$$
\begin{gathered}
T_{1}=C_{D} \cdot I_{1}(t)+\left(C_{D}+C_{1}\right) \cdot X_{1} N_{1} \\
T_{2}=C_{D} \cdot I_{2}(t) \\
T_{3}=C_{D} \cdot I_{3}(t)+\left(C_{D}+C_{3}\right) \cdot X_{3} N_{3}
\end{gathered}
$$

where $\mathrm{N}_{3}=\mathrm{N}_{1} / 32.83$ is the number of teachers in population.

\subsubsection{Incremental Cost-Effectiveness ratio}

The corresponding ICER for this scenario is calculated using the same formula as in section 2.1.3 but now by using total cost $T=T_{1}+T_{2}+T_{3}$.

\subsubsection{Strategies for MDA}

Scenario V: The proposed strategy in this case will be that, only children and high-risk adults will be covered under MDA. This is a more practical approach since the location of delivering MDA will be same. From the current policy, the budget can be equivalent of treating $0.8 \mathrm{~N}_{1}$ number of individuals. Thus, the proportion $X_{1}$, representing coverage level in children group, is varied in the range [0.0, 0.78] and the corresponding proportion of $X_{3}$, representing coverage level in high-risk group, is also the set of exact same values since we assume that the student-teacher ratio is fixed and constant. Hence, the relation $X_{1} N_{1}+X_{3} N_{3} \leq 0.8 N_{1}$ always holds for this case.

The identification of optimal coverage level in Ghana is carried out in two major steps: (i) the best strategy within each of the five scenarios is computed, and (ii) the best strategy from each scenario is compared with best strategy from other scenarios. Note, the best strategy for a scenario provides optimal coverage level based on age- or risk-structured populations under limited and unlimited budget constraints.

\subsection{Parameter estimates}

Initial prevalence in children is stated to be between 0.2 and 0.3 (Ministry of Health in Ghana, 2013). We choose an average value of 0.25 as prevalence for children. Prevalence in adults is estimated to be 0.20 (Campbell et.al, 2018)[25]. We use these values to estimate the value of $k$ (negative binomial aggregation parameter) using Equation $(2,3)$ derived from Equation (1). Ghana aims to "reduce morbidity related to STH to levels of no public health significance" [5]. The density-dependent coefficient $z$ which was set at 0.98 [17]. R (basic reproduction number) of 2 is taken in order to be conservative in value estimation [20]. All the other estimates can be found in Table 2. The values corresponding to children are averages of values of parameters corresponding to ages 0 to 14 . The values corresponding to worms are averages of all three types of worms.

$\mathrm{C}_{\mathrm{A}}$ is approximately $\$ 1.62$ assuming that it would cost 3 times the cost of school-based MDA [17] due to the logistical difficulties that may arise from traveling to different towns across Ghana. There may be the need to travel to some towns repeatedly due to superstitious beliefs regarding unorthodox medicines in order to convince residents of those towns to take the drug administered. So, all of these situations are taken into consideration in $\$ 1.62$ value. The estimates and sources for other cost model related variables is given in Table 3. The number of high-risk adults in the population $\left(\mathrm{N}_{3}\right)$ is calculated using the pupil-to-teacher ratio (32.83) in Ghana in 2016 according to the United Nations Educational, Scientific, and Cultural Organization (UNESCO) Institute for Statistics. 


\section{Results}

\subsection{Homogeneous mixing of adults with children}

Scenario I: The prevalence when there is no MDA, $P_{0}$, is obtained from World Health Organization, 2006 to be equal to 0.578 . The corresponding cost incurred in treating the infected population was calculated as $T_{0}=C_{D} I_{0}$ where $I_{0}=P_{0} N$. These values would serve as the baseline values for ICER for all potential strategies.

Scenario II: From Figure 7, it can be seen that the current policy in Ghana of treating $80 \%$ of the children with school-based MDA, it is possible to bring the prevalence down by a little but not low enough. Thus, we see that this policy would not be effective enough in the long-run and it would be almost impossible to reach a point where prevalence is below $1 \%$ like WHO aims for. And therefore, we set up the Scenarios III and IV to address this problem.

\section{Scenario III}
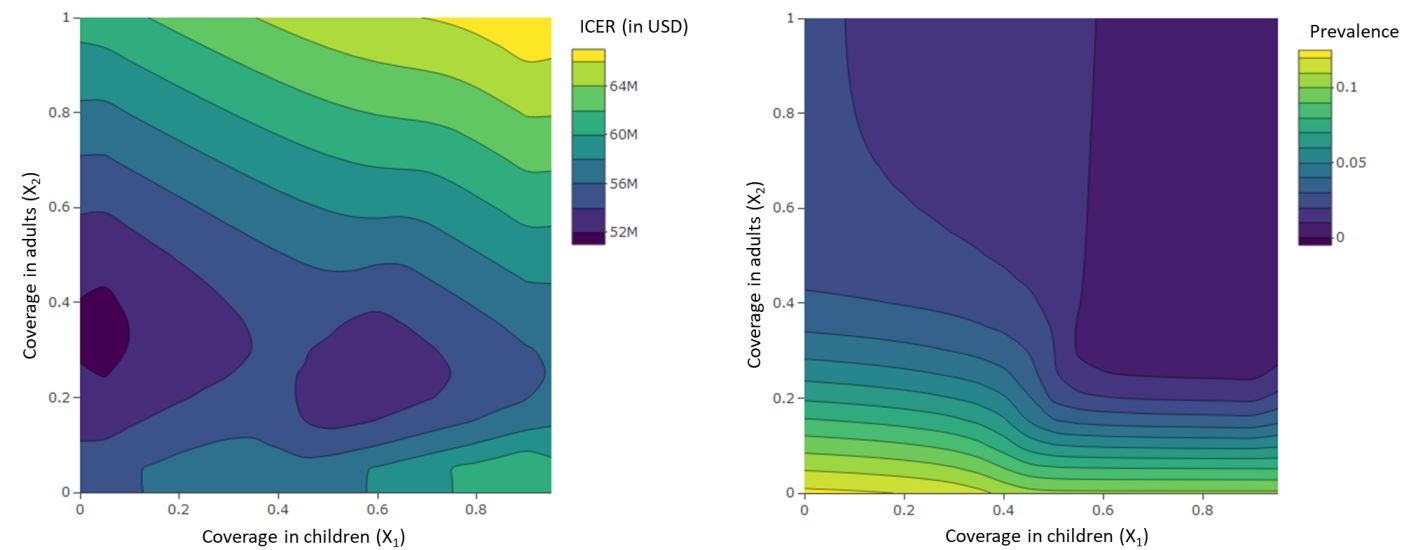

Figure 4. Changes in ICER (measure for cost-effectiveness) of MDA and corresponding model prevalence with varying coverages in children $\left(X_{1}\right)$ and adults $\left(X_{2}\right)$ assuming unlimited resources (Scenario III).

Scenario III: With the assumption of unlimited resources and budget, we tested for every possible combination of coverages in children and adults. Using the steady state prevalences from the simulated dynamical system, we calculate the ICER as described in previous section. Figure 4 shows the distribution of ICER and prevalence for all combinations of coverages of MDA in children and adults. From ICER solely, it could have been said that the best strategy would be to treat very less percentage of children but we can see that the prevalence for that strategy is higher. Thus, from both plots in Figure 4 we choose the strategy of covering $55 \%$ of the children and $25 \%$ of the adults such that it has lowest ICER in the region where prevalence is the least.

Scenario IV: When the number of individuals covered in MDA is fixed, that is when $X_{1} N_{1}+$ $X_{2} N_{2}=0.8 N_{1}$, the distribution of ICER can be seen in Figure 5. The $x$-axis is the proportion of coverage in adults and from the formula in previous line, the corresponding coverage in children can be calculated. From the results we find that the best strategy in this case would be to cover approximately 19\% of adults and approximately 50\% of children. Figure 7 shows how it reduces prevalence over time.

\subsection{Heterogeneous mixing}

Scenario V: When heterogeneous mixing in populations is considered, we split the adult population into low-risk and high-risk based on the likelihood of them mixing with children. This case assumes budget constrains which are implemented by limiting the number of individuals covered under MDA. The budget constrains in our model means that total number of individuals 
that can be covered under proposed MDA policy has to be same as under the current policy of the Ghana government, which considers a fixed number of school-aged children for MDA. Figure 6 shows changes in the prevalence of STH in Ghana as MDA coverage in high-risk adults changes. The prevalence steeply decreases at around high-risk group coverage of 0.4 and drops drastically until approximately 0.6 . Hence, it suggests that if $60 \%$ of high-risk group individuals were covered under MDA, the prevalence would see major decrease. Thus, the best strategy in this case would be to achieve $\sim 60 \%$ children and $\sim 60 \%$ high-risk adults. The comparison of different scenarios is shown in Figure 7. The result suggests that scenario V strategy gives almost the same behavior as the best strategy, Scenario II. Although the ICER increases linearly over the increasing proportions of coverage, ICER for Scenario V strategy is about 30\% less than ICER for Scenario II best strategy.

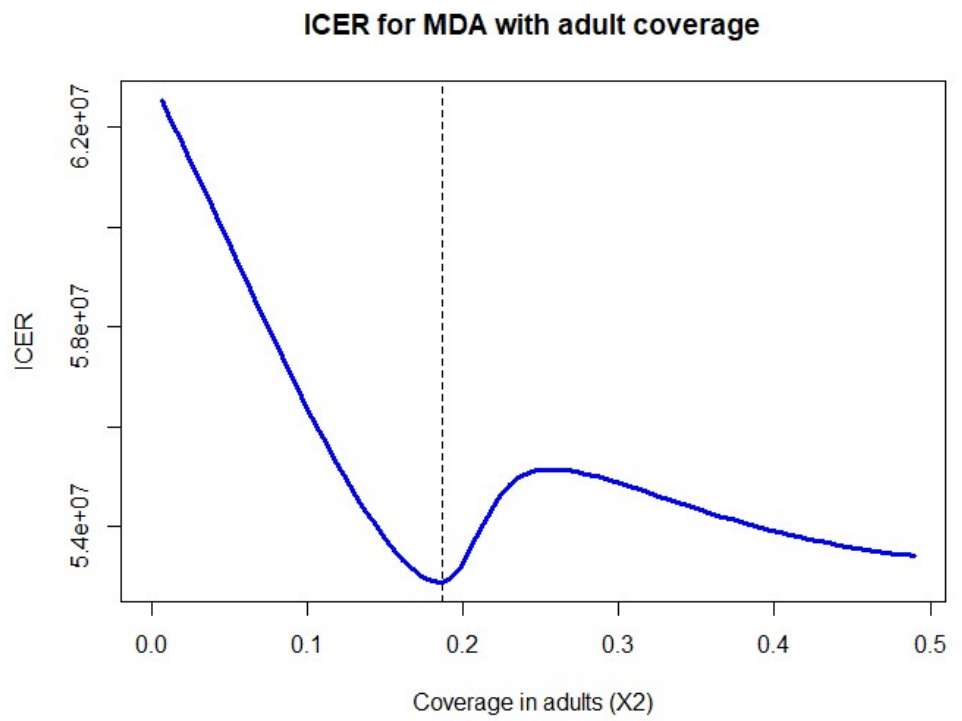

Figure 5. Cost-effectiveness in MDA with varying coverages in adults and children so that the total number of individuals covered in MDA is fixed (Scenario IV).

Prevalence of STH in Ghana upon risk-based MDA coverage

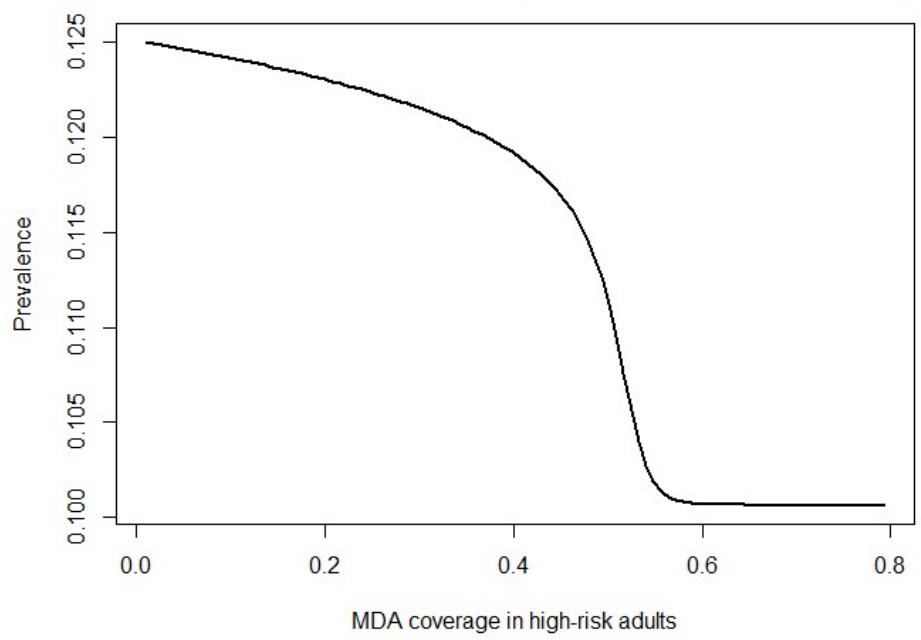

Figure 6. Prevalence of STH in Ghana based on different proportions of MDA coverages in the two groups, children and high-risk adults. 


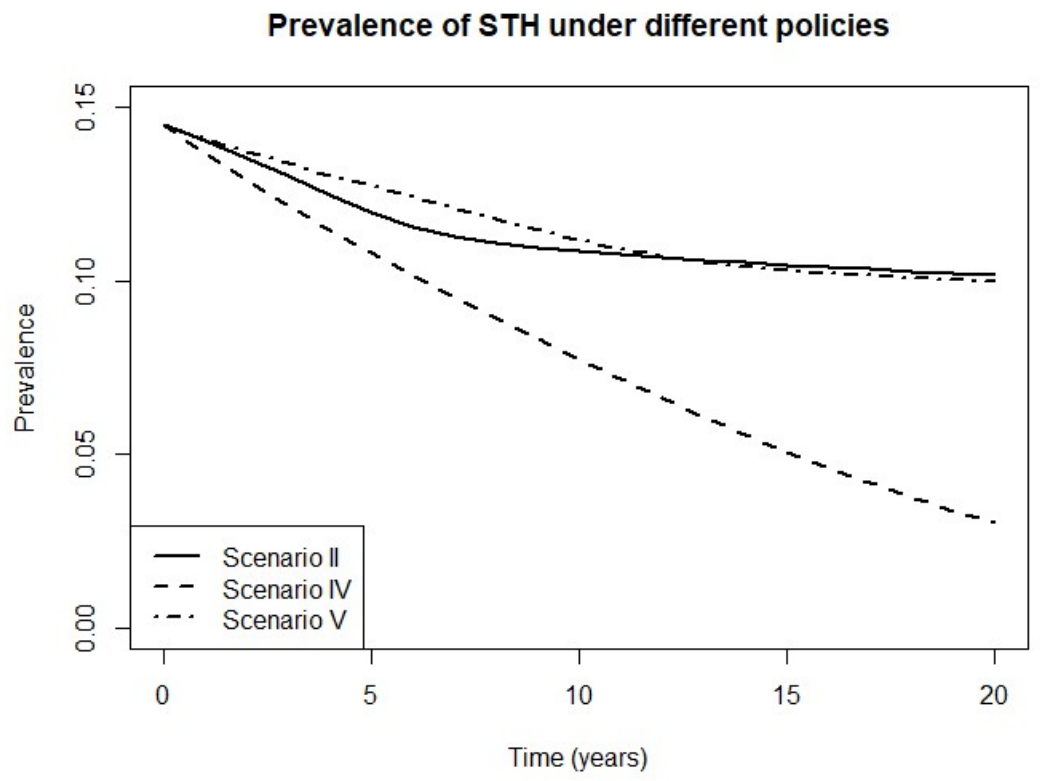

Figure 7. Prevalence of STH in Ghana over time upon implementation of different MDA coverage policies based on age (children and adults) and based on risk (children and only high-risk adults). Current policy corresponds to $\mathrm{X}_{1}=0.8, \mathrm{X}_{2}=0.0$, age-based policy is $\mathrm{X}_{1}=0.5, \mathrm{X}_{2}=0.19$ and risk-based policy is $\mathrm{X}_{1}=0.6, \mathrm{X}_{3}=0.6$.

\section{Discussion}

Soil-transmitted helminths (STH) is a major health problem in Ghana. Mass drug administration (MDA) is Ghana's primary control policy. MDA among children has limited efficacy due to the focus being on school-aged children only. The recommended coverage for MDA after a test for residual prevalence of MDA is greater than $75 \%$ in the population [26] since the focus of MDA is only on one age group, the recommended level of MDA is not been able to achieve and hence STH has been persisting at high level in Ghana. In this study we examine what percentages of coverage in adults and children would reduce prevalence within given budget. Additionally, we examine whether it is indeed cost-effective to conduct community-wide MDA that targets both adults and children as suggested by Lo et. al. (2015) within the Ghanaian context. Furthermore, we also evaluated the impact of policy, implementing MDA in high-risk adult (those that spend more time with children) population rather than focusing on adult individuals at random, on the prevalence of STH in Ghana.

Table 5. Summary of ICERs for the best strategies in each scenario as compared to no MDA.

\begin{tabular}{ccc}
\hline Scenario & Coverages & $\begin{array}{c}\text { ICER (In USD) } \\
\text { (Against Scenario I) }\end{array}$ \\
\hline $\begin{array}{c}\text { II: Current policy } \\
\text { III: Age-structured: Unlimited } \\
\text { budget }\end{array}$ & $\mathrm{X}_{1}=0.80, \mathrm{X}_{2}=0.00$ & 7096101 \\
$\begin{array}{l}\text { IV: Age-structured: Limited } \\
\text { number of individuals under } \\
\text { MDA }\end{array}$ & $\mathrm{X}_{1}=0.55, \mathrm{X}_{2}=0.25$ & 52382320 \\
$\begin{array}{c}\text { V: Risk-based: Limited number } \\
\text { of individuals under MDA }\end{array}$ & $\mathrm{X}_{1}=0.60, \mathrm{X}_{3}=0.19$ & 52707569 \\
\hline
\end{tabular}


It is known, MDA is crucial to control the neglected tropical disease STH. We use dynamics model and data from Ghana to address our research questions under different age-structured and budget-constrained scenarios. Scenario I captures the logistics when there is no MDA. In Scenario II when only children are treated, the prevalence rate is at its highest when there is no MDA in children and the prevalence decreases over time but not enough to get close to the WHO's target. Children will still get re-infected with the disease due to poor environmental conditions and due to transmission from pregnant women to their newborns. Infection and reinfection become cyclical. It was also seen that prevalence over time stabilizes at a value which is "not of public health significance" by WHO standards. Therefore, eradication in total population would be almost impossible when only children are targeted for mass drug administration. This emphasizes on the need for investigation of new strategies.

When adults are included in MDA, with the availability of unlimited resources and budget, we find that approximately $25 \%$ of adults and $55 \%$ of children seem to be the most cost-effective. This is in favor of the hypothesis that there should be at lekast some coverage of MDA in adults in addition to the MDA for school-age children. This is in line with the conclusion of Lo et. al. (2015) which focused on Ivory Coast. But the assumption of unlimited budget is highly unrealistic and therefore we further decide to analyze only a subset of it such that the number of individuals in MDA is fixed and finite. And that fixed number of individuals would be equal to $80 \%$ of children in Ghana $\left(0.8 N_{1}\right)$. This condition would make sure that the budget is approximately be equivalent to the cost incurred due to current policy. Thus in Scenario IV since ICER, the cost incurred against extra cases avoided, for $X_{1}=0.8, X_{2}=0.0$ was greater than many other combinations, it is clear that the current policy is not cost-effective. Based on this scenario, the best strategy is considering $\sim 19 \%$ adults and $\sim 50 \%$ children under MDA [27].

Lastly, a model for heterogeneous mixing of adults with children was developed and a scenario based on this model is analyzed. This scenario (Scenario $V$ ) is studied because there is high interaction of particular group of adults, like teachers, who are exposed more to the worms due to mixing disproportionately more with children rather than adults who interact or share space with only the children who are their kids. In Scenario $V$, we found that if the number of individuals under MDA has to be limited, $\sim 60 \%$ children and $\sim 60 \%$ high-risk adults needs to be covered to achieve drastic reduction in prevalence of STH. This strategy of scenario $\mathrm{V}$ gives the prevalence similar to the prevalence for current policy (Scenario II). Although the ICER for this strategy is not the lowest for different combinations in Scenario $V$, the ICER value in this strategy (60\% of children and high-risk adults each) is the lowest among the strategies for all other scenarios discussed above (Table 5). When we compare the ICERs for Scenario II and Scenario V specifically, it turns out that the ICER for former is about $30 \%$ higher. That is, same outcome can be achieved at a much lower cost if the teachers and school workers are also covered under MDA. To improve the outcomes further, based on the availability of resources, some coverage in low-risk adults can also be thought of.

The reasoning behind the hypothesis that adults should also be covered in MDA is that although in many areas, children are most vulnerable to this disease, the presence of the disease in adults such as pregnant women who end up passing the worms to their unborn children will increase the spread of the disease. Adult farmers such as tea-pickers who are also susceptible to this disease can pick it up in soils in temperate regions [28]. Reinfection by adults promotes the spread of the disease and betrays the purpose of reduction of transmission of STH through mass drug administration as recommended by the WHO. Reducing transmission in adults could reduce prevalence in pre-SAC (newborns and children between the ages of 0-4 years) and prevalence overall. However, the MDA should be focused on high-risk adults rather than randomly chosen adults. In addition, Disability Adjusted Life Years "DALY dollars" can be averted to reduce the amount of money lost due to the inability of adults to work and contribute to the economy when included in MDA efforts.

Finally, we report some limitations of our models and results. Even though we estimate the proportions of coverage for the most cost-effective policy, we would like to remark that these results are based on the dynamical model whose some parameter estimates for Ghana may not be precise. It 
is noted that the results for prevalence change may depend on parameters such as the contact rates between different groups in a population and the total MDA implementation cost, which depends on individual costs of human resources and delivery specific to the region of focus. Furthermore, the costs incurred over time also need to be collected to get a better validation of the cumulative efforts to control of the disease. In spite of some of these limitations, this study provides the first estimate of MDA's cost-effective strategies and coverage levels for the STH in Ghana.

In conclusion, implementing MDA in only children does reduce the disease prevalence but reduction of STH to levels of no public significance may not be possible. However, implementing MDA in at least some proportion of adults reduces prevalence faster and effectively. There might be some challenges in randomly finding and delivering MDA to adults for MDA, hence, it will be more feasible to focus on high-risk adults who can be more approachable through existing delivery mechanisms of MDA such as school teachers or workers. In addition, reduction in morbidity to a level of no public health significance as aimed by the Ministry of Health could be achieved by systematically focusing on both adult and children group rather than focusing on only one of the groups.

This work also gives insight on the need for measurement of Ghana-specific parameters to get more realistic estimates of the combination of the coverages that can significantly bring the disease prevalence down. QALY and DALY metrics need to be researched in the Ghanaian context in order to make accurate estimations of costs and intervention. Our models assumed that there is a cost associated with albendazole drug used in MDA rather than it being donated to public health department, the cost of community-based MDA targeted to adults is 3 times the cost of school-based MDA, the cost of implementation is the sum of the cost of only human resources and transportation are incurred and the cost of implementing MDA per patient in both groups decreases as coverage is increased. The assumptions may be varied to study uncertainty in the model implications.

To improvise the results further, the dynamical model for heterogeneous mixing can be extended to incorporate the coverage of low-risk adults too. The framework of this research study is important because it sheds lights on the use of mathematical models as tools that are critical in understanding the impact of health services such as mass drug administration. Additionally, this study suggests that community-wide mass drug administration at optimal percentage of coverage with consideration of the above-mentioned case setups is imperative in the reduction of prevalence of STH in Ghana. Researches on cost analysis to understand incremental utility and benefits are needed in Ghana in order to better understand the policies and actions that underpins Ghana's health structure.

Author Contributions: Conceptualization, Methodology, Writing- review and editing: A.M., M.T.; Resources, Writing- Original draft: R.M.; Project Administration, Supervision: A.M.; Formal Analysis, Visualization: M.T.

Funding: R.M. was funded by MasterCard Foundation Scholars' Program. A.M. and M.T. were partly funded by NSF-DMS-1840884.

Acknowledgments: R.M. would like to acknowledge Dr. Popova (Arizona State University, USA) for her guidance. We would like to thank Dr. Bartoszczuk (Warsaw School of Economics, Poland) for is guidance on cost-analysis. We would like to acknowledge Dr. Castillo-Chavez (Arizona State University, USA) for his input on the heterogeneity in the population.

Conflicts of Interest: The authors declare no conflict of interest. The funders had no role in the design of the study; in the collection, analyses, or interpretation of data; in the writing of the manuscript, or in the decision to publish the results.

\section{Appendix A: Health metrics}

Cost-Effectiveness Analysis: Cost-effectiveness analysis is used to assess benefits in a health service relative to the costs of alternative health interventions. It helps in identifying "neglected opportunities by highlighting interventions that are relatively inexpensive yet have the potential to reduce the disease burden substantially" [29]. Cost-effectiveness analysis (CEA) helps policymakers in determining where to redirect the already limited resources so as to make the most gains. 
Additionally, it helps in determining which health interventions is more effective between quantity of life and quality of life at a certain cost. According to the World Health Organization, "while cost is measured in monetary terms, effectiveness is determined independently and may be measured in terms of a clinical outcome such as number of lives saved, complications prevented or diseases cured... and it measures the incremental cost of achieving an incremental health benefit expressed as a particular health outcome" [5]. Examples of Incremental Cost Effectiveness Ratios (ICERs) using CEA are the cost per additional patient attaining a $10 \mathrm{~mm} \mathrm{Hg}$ fall in blood pressure and the cost per additional asthmatic patient attaining a reduction in oral corticosteroid use [5]. CEA can also be expressed in DALY which stands for Disability-Adjusted Life Year. It is a "universal metric that allows researchers and policymakers to compare very different populations and health conditions across time... one DALY equals one lost year of healthy life. DALYs allow us to estimate the total number of years lost due to specific causes and risk factors at the country, regional, and global levels" [30]. DALYs lost due to morbidity (the condition of being sick) inadvertently leads to loss of productive hours; in adults that means reduction in hours that they could have spent working to contribute to the economy of the country. DALY in West-Africa was estimated to be about $13.8 \%$ of the worldwide DALY of 5.18 million for all three species of helminths [31]. This means that about 715,000 life years are lost annually due to mortality and morbidity of this disease and most of the life years lost are unsurprisingly concentrated in developing countries. Ghana, a low-income country in West-Africa is of no exception. Fortunately, drugs that are needed for the treatment of STH are sometimes donated but Jourdan et. al 2018 add that "although safe and effective drugs are donated free to endemic countries, only half of at-risk children received treatment in 2016" [12]. Cost-Effective Analysis "is relatively easy to undertake and the benefits are measured as a single unidimensional outcome; however, other potentially important outcomes may be ignored" [22]. Due to its unidimensionality, there may be errors in conclusions made using CEA. Nevertheless, CEA is very instrumental in providing estimates in cost-effectiveness that inform healthcare decision-making process.

Cost-Utility Analysis: Cost-utility Analysis (CUA) is the measure of both the quantity and the quality of life gained. It is used to determine cost in terms of utilities, especially quantity and quality of life [5]. This type of cost analysis is used to compare two different interventions or two different drugs in order to express the monetary value of a single health outcome. The Incremental Cost-Effectiveness Ratio (ICER) in this case is valued at the incremental cost when 1 extra Quality-Adjusted Life Year (QALY) is gained through a treatment such as MDA. This analysis is controversial because there are diverging opinions on the definition of quality of life or the value of life gained. Quality of life is measured on a scale of 0 (dead) to one (great quality of life). CUA is quantified as life years gained.

Cost-Benefit Analysis: According to the World Health Organization, "cost-benefit analysis is used to value both incremental costs and outcomes in monetary terms and therefore allows a direct calculation of the net monetary cost of achieving a health outcome" [5]. Life year gained could be valued in terms of average wage gained in the year. Cost-benefit analyses can be trial based or modelled. ICER for trial-based analyses is calculated with the use of incremental benefits and resources in a clinical trial condition. Modelled analyses are used to apply the benefits and use of resources both to a local clinical situation, and also to extend the time frame of a clinical trial. This according to the World Health Organization "is particularly important where the benefits of treatment may not be realized until sometime in the future; two examples are the avoidance of liver cancer or transplantation for patients with hepatitis $C$ and the prolongation of life for hypertensive patients" [5]. 


\section{References}

1. Hotez, P.J., Bundy, D.A.P., Beegle, K., Brooker, S., Drake, L., de Silva, N., Montresor, A., Engels, D., Jukes, M., and Chitsulo, L. "Helminth Infections: Soil-Transmitted Helminth Infections and Schistosomiasis." (2006).

2. Hotez, P. "Hookworm and Poverty." J Annals of the New York Academy of Sciences 1136, no. 1 (2008): 38-44.

3. Adu-Gyasi, D., Asante, K.P., Frempong, M.T., Gyasi, D.K., Iddrisu, L.F., Ankrah, L., Dosoo, D., Adeniji, E., Agyei, O., and Gyaase, S. "Epidemiology of Soil Transmitted Helminth Infections in the Middle-Belt of Ghana, Africa." J Parasite Epidemiology Control 3, no. 3 (2018).

4. "Parasites- Hookworm." https://www.cdc.gov/parasites/hookworm/biology.html (accessed 15 February 2018).

5. "Soil-Transmitted Helminths Infection." http:/www.who.int/mediacentre/factsheets/fs366/en/ (accessed 8 April 2018).

6. Loukas, A., Hotez, P.J., Diemert, D., Yazdanbakhsh, M., McCarthy, J.S., Correa-Oliveira, R., Croese, J., and Bethony, J.M. "Hookworm Infection." J Nature Reviews Disease Primers 2 (2016): 16088.

7. Webster, J.P., Molyneux, D.H., Hotez, P.J., and Fenwick, A. "The Contribution of Mass Drug Administration to Global Health: Past, Present and Future." J Phil. Trans. R. Soc. B 369, no. 1645 (2014): 20130434.

8. Keenan, J.D., Hotez, P.J., Amza, A., Stoller, N.E., Gaynor, B.D., Porco, T.C., and Lietman, T.M. "Elimination and Eradication of Neglected Tropical Diseases with Mass Drug Administrations: A Survey of Experts." J PLoS neglected tropical diseases 7, no. 12 (2013): e2562.

9. Farrell, S.H., Truscott, J.E., and Anderson, R.M. "The Importance of Patient Compliance in Repeated Rounds of Mass Drug Administration (Mda) for the Elimination of Intestinal Helminth Transmission." J Parasites vectors 10, no. 1 (2017): 291.

10. "Ghana Neglected Tropical Disease Masterplan 2013-2017." In Ministry of Health in Ghana, 2013.

11. Smits, H.L. "Prospects for the Control of Neglected Tropical Diseases by Mass Drug Administration." J Expert review of anti-infective therapy 7, no. 1 (2009): 37-56.

12. Jourdan, P.M., Lamberton, P.H.L., Fenwick, A., and Addiss, D.G. "Soil-Transmitted Helminth Infections." J The Lancet 391, no. 10117 (2018): 252-65.

13. Anderson, R.M., and May, R.M. "Population Dynamics of Human Helminth Infections: Control by Chemotherapy." J Nature 297, no. 5867 (1982): 557.

14. Anderson, R.M., and May, R.M. "Infectious Diseases of Humans." J Oxford University Press 1 (1991): 188.

15. Moser, W., Schindler, C., and Keiser, J. "Efficacy of Recommended Drugs against Soil Transmitted Helminths: Systematic Review and Network Meta-Analysis." J bmj 358 (2017): j4307.

16. Truscott, J., Turner, H., Farrell, S., and Anderson, R. "Soil-Transmitted Helminths: Mathematical Models of Transmission, the Impact of Mass Drug Administration and Transmission Elimination Criteria." In Advances in Parasitology, 133-98: Elsevier, 2016.

17. Lo, N.C., Bogoch, I.I., Blackburn, B.G., Raso, G., N'Goran, E.K., Coulibaly, J.T., Becker, S.L., Abrams, H.B., Utzinger, J., and Andrews, J.R. "Comparison of Community-Wide, Integrated Mass Drug Administration Strategies for Schistosomiasis and Soil-Transmitted Helminthiasis: A Cost-Effectiveness Modelling Study." J The Lancet Global Health 3, no. 10 (2015): e629-e38. 
18. Anderson, R.M., Truscott, J.E., Pullan, R.L., Brooker, S.J., and Hollingsworth, D.T. "How Effective Is School-Based Deworming for the Community-Wide Control of Soil-Transmitted Helminths?" J PLoS neglected tropical diseases 7, no. 2 (2013): e2027.

19. Turner, H.C., Toor, J., Hollingsworth, D.T., and Anderson, R.M. "Economic Evaluations of Mass Drug Administration: The Importance of Economies of Scale and Scope." J Clinical Infectious Diseases 66, no. 8 (2017): 1298-303.

20. Truscott, J.E., Hollingsworth, D.T., Brooker, S.J., and Anderson, R.M. "Can Chemotherapy Alone Eliminate the Transmission of Soil Transmitted Helminths?" J Parasites vectors 7, no. 1 (2014): 266.

21. "Essential Medicines and Health Products." (accessed 30 August 2018).

22. "An Introduction to the Principles of Critical Appraisal of Health Economic Evaluation Studies." https://www.nlm.nih.gov/nichsr/edu/healthecon/04_he_06.html (accessed 30 March 2018).

23. "The Cost of Large-Scale School Health Programmes Which Deliver Anthelmintics to Children in Ghana and Tanzania." J Acta Tropica 73, no. 2 (1999): 183-204.

24. Turner, H.C., Osei-Atweneboana, M.Y., Walker, M., Tettevi, E.J., Churcher, T.S., Asiedu, O., Biritwum, N.-K., and Basáñez, M.-G. "The Cost of Annual Versus Biannual Community-Directed Treatment of Onchocerciasis with Ivermectin: Ghana as a Case Study." J PLoS neglected tropical diseases 7, no. 9 (2013): e2452.

25. Campbell, S.J., Osei-Atweneboana, M.Y., Stothard, R., Koukounari, A., Cunningham, L., Armoo, S.K., Biritwum, N.-K., Gyapong, M., MacPherson, E., and Theobald, S. "The Countdown Study Protocol for Expansion of Mass Drug Administration Strategies against Schistosomiasis and Soil-Transmitted Helminthiasis in Ghana." J Tropical Medicine Infectious Disease 3, no. 1 (2018): 10.

26. Bradbury, R.S., and Graves, P.M. "Current Who Protocols for Mass Drug Administration in Helminth Control." J Microbiology Australia 37, no. 1 (2016): 10-12.

27. Tajebe, F., Getahun, M., Adem, E., Hailu, A., Lemma, M., Fikre, H., Raynes, J., Tamiru, A., Mulugeta, Z., Diro, E., Toulza, F., Shkedy, Z., Ayele, T., Modolell, M., Munder, M., Müller, I., Takele, Y., and Kropf, P. "Disease Severity in Patients with Visceral Leishmaniasis Is Not Altered by Co-Infection with Intestinal Parasites." PLOS Neglected Tropical Diseases 11, no. 7 (2017): e0005727.

28. Turner, H.C., Truscott, J.E., Hollingsworth, D.T., Bettis, A.A., Brooker, S.J., and Anderson, R.M. "Cost and Cost-Effectiveness of Soil-Transmitted Helminth Treatment Programmes: Systematic Review and Research Needs." J Parasites vectors 8, no. 1 (2015): 355.

29. Jamison, D.T., Breman, J.G., Measham, A.R., Alleyne, G., Claeson, M., Evans, D.B., Jha, P., Mills, A., and Musgrove, P. Disease Control Priorities in Developing Countries: The World Bank, 2006.

30. "What Is Daly." http://www.healthdata.org/gbd/faq (accessed April 4 2018).

31. Pullan, R.L., Smith, J.L., Jasrasaria, R., and Brooker, S.J. "Global Numbers of Infection and Disease Burden of Soil Transmitted Helminth Infections in 2010." J Parasites vectors 7, no. 1 (2014): 37. 